\title{
Erratum
}

\section{Stereocontrolled Synthesis of Enantiopure Polyhydroxylated Azetidines via 1,2-Oxazines}

Vjekoslav Dekaris, Hans-Ulrich Reissig* Synlett 2010, 42.

The formulas for compounds anti-1b and $\mathbf{2 c}$ as drawn in Table 1 are wrong. The 4-OBn group in these compounds should be replaced by a OMe group. The formula of $\mathbf{2 c}$ as given in Table 2 is correct. The authors apologize for this mistake. 